\title{
Differential Expression of In Vivo and In Vitro Protein Profile of Outer Membrane of Acidovorax avenae Subsp. avenae
}

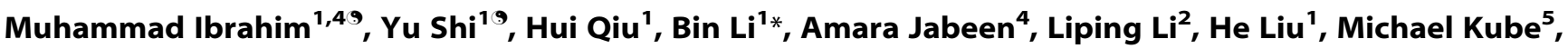 \\ Guanlin Xie', Yanli Wang ${ }^{3}$, Guochang Sun ${ }^{3}$
}

1 State Key Laboratory of Rice Biology, Institute of Biotechnology, Zhejiang University, Hangzhou, China, 2 Institute of Bioinformatics, Zhejiang University, Hangzhou, China, 3 State Key Laboratory Breeding Base for Zhejiang Sustainable Pest and Disease Control, Institute of Plant Protection and Microbiology, Zhejiang Academy of Agricultural Sciences, Hangzhou, China, 4 Department of Biosciences, COMSATS Institute of Information Technology, Sahiwal, Pakistan, $\mathbf{5}$ Faculty of Agriculture and Horticulture, Humboldt-Universität zu Berlin, Berlin, Germany

\begin{abstract}
Outer membrane (OM) proteins play a significant role in bacterial pathogenesis. In this work, we examined and compared the expression of the OM proteins of the rice pathogen Acidovorax avenae subsp. avenae strain RS-1, a Gram-negative bacterium, both in an in vitro culture medium and in vivo rice plants. Global proteomic profiling of A. avenae subsp. avenae strain RS-1 comparing in vivo and in vitro conditions revealed the differential expression of proteins affecting the survival and pathogenicity of the rice pathogen in host plants. The shotgun proteomics analysis of OM proteins resulted in the identification of 97 proteins in vitro and 62 proteins in vivo by mass spectrometry. Among these OM proteins, there is a high number of porins, TonB-dependent receptors, lipoproteins of the NodT family, ABC transporters, flagellins, and proteins of unknown function expressed under both conditions. However, the major proteins such as phospholipase and OmpA domain containing proteins were expressed in vitro, while the proteins such as the surface anchored protein F, ATPdependent Clp protease, OmpA and MotB domain containing proteins were expressed in vivo. This may indicate that these in vivo OM proteins have roles in the pathogenicity of $A$. avenae subsp. avenae strain RS-1. In addition, the LC-MS/MS identification of OmpA and MotB validated the in silico prediction of the existance of Type VI secretion system core components. To the best of our knowledge, this is the first study to reveal the in vitro and in vivo protein profiles, in combination with LC-MS/MS mass spectra, in silico OM proteome and in silico genome wide analysis, of pathogenicity or plant host required proteins of a plant pathogenic bacterium.
\end{abstract}

Citation: Ibrahim M, Shi Y, Qiu H, Li B, Jabeen A, et al. (2012) Differential Expression of In Vivo and In Vitro Protein Profile of Outer Membrane of Acidovorax avenae Subsp. avenae. PLoS ONE 7(11): e49657. doi:10.1371/journal.pone.0049657

Editor: Raymond Schuch, Rockefeller University, United States of America

Received June 27, 2012; Accepted October 11, 2012; Published November 15, 2012

Copyright: (C) $2012 \mathrm{lbrahim}$ et al. This is an open-access article distributed under the terms of the Creative Commons Attribution License, which permits unrestricted use, distribution, and reproduction in any medium, provided the original author and source are credited.

Funding: This project was supported by Zhejiang Provincial Natural Science Foundation of China (Y3090150), Zhejiang Provincial Project (2010R10091), the Fundamental Research Funds for the Central Universities, the Agricultural Ministry of China (nyhyzx 201003029; 201003066), State Education Ministry and Key Subject Construction Program of Zhejiang for Modern Agricultural Biotechnology and Crop Disease Control (2010DS700124-KF1101). The funders had no role in study design, data collection and analysis, decision to publish, or preparation of the manuscript.

Competing Interests: The authors have declared that no competing interests exist.

*E-mail: libin0571@zju.edu.cn

9 These authors contributed equally to this work.

\section{Introduction}

Acidovorax avenae subsp. avenae is a phytobacterium that can cause diseases in many economically important plants, including rice, corn, oats, sugarcane, millet, and foxtail [1]. In particular, rice strains of $A$. avenae subsp. avenae have caused severe losses in rice throughout many countries in Asia, Africa, the Americas and Europe $[1,2,3]$. The whole genome of $A$. avenae subsp. avenae strain RS-1 from rice has been fully sequenced in our previous study [1], which is a useful resource to identify genes involved in some specific biological functions such as the interaction between the pathogen $A$. avenae subsp. avenae and its rice host.

The interaction of bacterial pathogens with host cells is closely related to the expression of OM proteins, which are structurally distinct from those that reside in the inner membrane [4]. Indeed, some OM proteins from different Gram-negative bacteria not only have been recognized as important virulence factors and targets for host immune recognition [5], but also have recently been proposed to be required for the bacterial Type VI secretion system (T6SS). This represents a new paradigm of protein secretion that is critical for the pathogenesis of many Gram-negative bacteria [6].

Identification of abundant and/or novel OM proteins, in particular OM components of the T6SS, and characterization of their roles in pathogen physiology, disease, and defense against the host, is an important preliminary step in understanding the pathogenesis [5]. Recently, a variety of studies have investigated the OM proteome of Gram-negative bacteria including Xanthomonas campestris, E. coli, Aeromonas salmonicida, Proteus mirabilis, Burkholderia pseudomallei, Dickeya dadantii, Burkholderia mallei, Yersinia pestis, Actinobacillus pleuropneumoniae and Bartonella henselae $[5,7,8,9,10]$.

Most of this research focused on the proteome of in vitro cultivated bacteria [11]. However, it is the characterization of the 
bacterial OM proteome and T6SS during in vivo infection of its host that eventually could provide the most significant insights into bacterial pathogenesis [12]. Indeed, proteomic analysis of OM proteins in human and animal pathogens such as Proteus mirabilis has revealed differential expression in vivo vs. in vitro conditions. In contrast, little is known about the in vivo expression profile of plant pathogenic bacteria due to the absence of an efficient method to obtain bacterial cells from plant tissue.

In this study, we developed a method to collect the in vivo bacteria from rice plants, which makes it possible to analyze in vivo OM proteome and the T6SS of $A$. avenae subsp. avenae strain RS-1. We present the in vitro vs. in vivo $\mathrm{OM}$ proteome profiling by using LC-MS/MS in combination with an in silico analysis of OM proteome and T6SS proteins of A. avenae subsp. avenae strain RS-1, while proteomic analysis of OM proteins revealed the differential composition between in vivo and in vitro. In particular, T6SS core components OmpA/MotB domain containing proteins and an ATP dependent Clp protease was identified in the OM proteome under in vivo conditions, but not under in vitro conditions. This validated the in silico prediction of the existance of T6SS core components or associated proteins and highlighted that several OM proteins may be involved in the survival and pathogenicity of A. avenae subsp. avenae strain RS-1 in host plants.

\section{Results and Discussion}

In this study, we applied a strategy combining the benefits of LC-MS/MS for a comprehensive coverage of proteins from host recovered bacteria with in silico predicted OM proteins and genome wide predicted T6SS proteins. Results of this study clearly revealed the differential expression of $\mathrm{OM}$ proteins in $A$. avenae subsp. avenae strain RS-1 between in vivo and in vitro. Indeed, a nonredundant list of $\mathrm{OM}$ proteins has been assembled in $A$. avenae subsp. avenae strain RS-1 by using LC-MS/MS and in silico prediction (Tables S1, S2). Of the proteins, 48 were identified as shared proteins under both in vitro and in vivo conditions (Table S3). However, 49 proteins were identified under in vitro but not in vivo (Table S4), while 14 proteins identified under in vivo but not in vitro (Table 1). Overall, the results confirmed our main hypothesis that the expression of OM proteins may play key roles in the interaction of bacterial pathogens with host cells and survival within the host cells.

\section{In vivo vs. in vitro $1 \mathrm{D}$ Profile of OM Proteins}

The in vitro and in vivo OM proteins of $A$. avenae subsp. avenae strain RS-1 were prepared as described in Materials and Methods and proteins were separated by 1D SDS-PAGE. As shown in Figure 1, the OM protein profiles of $A$. avenae subsp. avenae strain RS-1 in vivo are different from those of in vitro in general. Although, there is a high similarity in some protein bands between in vitro and in vivo. The protein bands that appeared by silver staining were cut from the gel into small pieces and were further digested with trypsin. The resultant peptides were then analyzed with the help of LC-MS/MS.

\section{Global Proteins Profiling of RS-1 Proteome}

LC-MS/MS profiles of the peptides from A. avenae subsp. avenae strain RS-1 were used for searching the sequence similarities to data from $A$. avenae or closely related Gram-negative bacteria available in the NCBI database. A proteome-wide dataset of proteins was compiled for putative protein localization in $A$. avenae subsp. avenae strain RS-1. Two subcellular localization predictor (PSORTb and Phobius) were used to assemble an inventory of potential OM proteins encoded in the genome of $A$. avenae subsp. avenae strain RS-1 and identified by LC-MS/MS. Of the total 5,043 proteins of $A$. avenae subsp. avenae strain RS-1 submitted to PSORTb and Phobius [13,14], 85 were predicted to be localized to the OM. In-gel tryptic digestion data (LC-MS/MS) from two biological replicates were in general consistent, and the combined data from two biological replicates revealed more than 97 putative OM proteins with $>98 \%$ confidence in vitro, while more than 62 putative OM proteins with $>97 \%$ confidence in vivo. After retrieving proteins identified under in vitro and in vivo condition and removing the overlapping protein entries and cytoplasmic proteins from each conditions, a non-redundant list of 159 proteins (in vitro and in vivo) was assembled and designated as OM as well as probable OM proteins in $A$. avenae subsp. avenae strain RS-1 by PSORTb and Phobius (Tables S1, S2). Of the 97 putative OM proteins under in vitro conditions, 63 proteins were among the list of $85 \mathrm{OM}$ proteins predicted by PSORTb and Phobius [13,14] and similarly, of the 62 putative proteins under in vivo conditions, 45 were among the list of 85 OM proteins predicted by PSORTb and Phobius $[13,14]$ in the genome of $A$. avenae subsp. avenae strain RS-1.

PSORTb and Phobius analysis [13,14] of the above identified $159 \mathrm{OM}$ and probable OM proteins showed that most of these proteins are homologous to well-documented $\mathrm{OM}$ associated proteins, such as TonB-dependent receptor, OM efflux protein, OmpA domain-containing protein and porin Gram-negative type, with high and medium confidence levels (Tables S1, S2). However, about $5 \%$ of all OM proteins, such as the LysR family transcriptional regulator, the gamma subunit of the $\mathrm{F}_{0} \mathrm{~F}_{1}$ ATP synthase, a phasin family protein and several so far uncharacterized proteins, were predicted to have multiple localization sites based on a statistical analysis of the SwissProt database, Phobius $[13,15]$ and PSORTb [14], and were included in the list of lipoproteins and other probable OM proteins (Tables S1, S2).

\section{Elucidation and Characterization of in vitro OM Proteome}

In total, 97 proteins were identified in the $\mathrm{OM}$ and probable OM proteome of $A$. avenae subsp. avenae strain RS-1 by LC-MS/ MS under in vitro conditions. After retrieving proteins identified under in vitro conditions and removing the overlapping protein entries, a non-redundant list of $63 \mathrm{OM}$ proteins remained from the whole list of OM proteins that had been predicted based on in silico analysis of $A$. avenae subsp. avenae strain RS-1 by PSORTb and Phobius (Table S1). Almost all of the $63 \mathrm{OM}$ proteins identified in strain RS-1 of $A$. avenae subsp. avenae had the highest sequence similarity with those of $A$. avenae subsp. avenae strain ATCG19860 with the exception of a few proteins showing the highest level of sequence similarity to other plant pathogens (Table S1). The detailed topology of LC-MS/MS identified OM proteins in A. avenae subsp. avenae strain RS-1 is as follows:

Twelve TonB-dependent receptor/complex proteins (TBDR) (COG1629) were identified in the $\mathrm{OM}$ of $A$. avenae subsp. avenae strain RS-1 (Table S1). This result is consistent with the result of Blanvillain et al. [16], who found that the OM of Gram-negative bacteria is enriched with TBDR, a family of beta-barrel proteins. However, the number of TBDRs in this study is about twice that of $B$. pseudomallei [5], which may be attributed to the difference in bacterial species. The TonB complex works to sense signals from outside the bacterial cell and transmits them via two membranes into the cytoplasm, leading to transcriptional activation of target genes [17]. TBDRs are also carbohydrate scavenger [16] and may play a key role in sensing and exploitation of plant derived carbon sources for A. avenae subsp. avenae. Furthermore, TonB is often involved in metal acquisition, especially iron, which is essential for 
Table 1. List of LC-MS/MS identified unique outer membrane protiens under in vivo condition of Acidovorax avenae subsp. avenae strain RS-1 proteome.

\begin{tabular}{|c|c|}
\hline Locus Tag & Unique outer membrane protiens in vivo \\
\hline Acav_0812 & TonB-dependent receptor/A. avenae subsp. avenae ATCC 19860 \\
\hline Aave_3310 & Binding domain containing prottein/Acidovorax citrulli AAC00-1 \\
\hline Aave_2079 & unnamed protein product/Acidovorax citrulli AAC00-1 \\
\hline Aave_4400 & phasin family protein/Acidovorax avenae subsp. avenae ATCC 19860 \\
\hline Aave_3309 & flagellin/Acidovorax citrulli AACO0-1 \\
\hline Acav_0496 & OmpA/MotB domain-containing protein/A. avenae subsp. avenae ATCC 19860 \\
\hline Acav_2010 & ATP-dependent Clp protease/A. avenae subsp. avenae ATCC 19860 \\
\hline Acav_3880 & General secretion pathway protein $\mathrm{F}$ \\
\hline Acav_4244 & porin Gram-negative type/A. avenae subsp. avenae ATCC 19860 \\
\hline Acav_4297 & flagellin domain-containing protein/A. avenae subsp. avenae ATCC 19860 \\
\hline Acav_4298 & flagellin/A. avenae subsp. avenae ATCC 19860 \\
\hline AcdelDRAFT_1224 & Phosphate $\mathrm{ABC}$ transporter PBP/Acidovorax delafieldii 2AN \\
\hline \multirow[t]{2}{*}{ Ajs_0682 } & porin Gram-negative type/Acidovorax sp. JS42 \\
\hline & Major OM lipoprotein/Pseudomonas oleovorans \\
\hline
\end{tabular}

doi:10.1371/journal.pone.0049657.t001

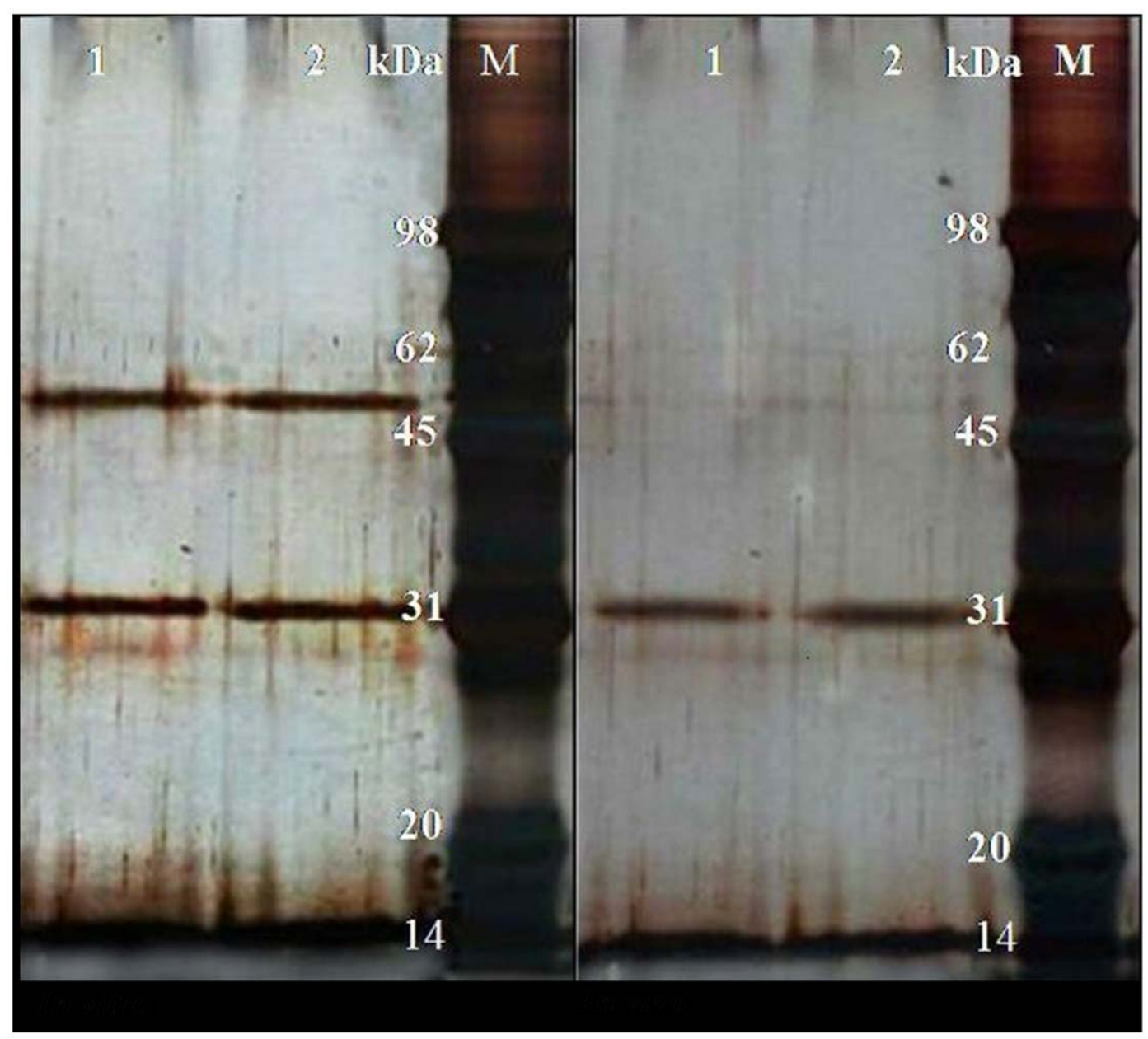

Figure 1. SDS-PAGE profile of Acidovorax avenae subsp. avenae strain RS-1 under in vivo and in vitro conditions. A) Represents the in vitro growth conditions where Lane $M$ represents the marker while Lane 1 and 2 represent the two replicates. B) Represents the in vivo growth conditions where Lane $M$ represents the marker while Lane 1 and 2 represent the two replicates.

doi:10.1371/journal.pone.0049657.g001 
all bacteria [17], and it also has been reported as an amylosucrase and a regulator of sucrose utilization [16].

Eleven proteins have been assigned as porins (COG3203), and have been presented in Table S1. They comprised the second populous group of $\mathrm{OM}$ proteins in $A$. avenae subsp. avenae strain RS-1 proteome. This result is consistent with previous studies that demonstrated that Gram-negative bacteria encode in high number porins $[5,10,18,19,20]$. Porins are beta barrel folded proteins that provide water-filled channels through which molecules can diffuse and allow the diffusion of hydrophilic molecules across the OM. They also can act as receptors for bacteriophages and are involved in a variety of functions including principal molecular determinant of phage susceptibility [21]. We have identified 5 OM efflux proteins (COG1538), which is a group of lipoproteins from the NodT family of the RND (Resistance-Nodulation-cell Division) type efflux systems that form trimeric channels with a $\beta$-barrel to allow export of diverse substrates including antibiotics [17,22]. These efflux proteins work with an inner membrane ABC transporter protein (ATPase subunit) and an adapter called a membrane fusion protein. Most of these efflux proteins are likely to export primarily small molecules rather than proteins and are related to the type I secretion OM proteins TolC and PrtF. The presence of NodT family of RND efflux pump in $A$. avenae subsp. avenae strain RS-1 may thus be functionally similar to those in $E$. coli and other Gram-negative bacteria, where they act as a major efflux system involved in the resistance of bacteria to a variety of toxic molecules, including antibiotics, dyes and detergents [18]. In addition, two members of the OmpW and OmpA families were also identified in the $\mathrm{OM}$ proteome of $A$. avenae subsp. avenae strain RS-1. These proteins are believed to play multifunctional roles but the mode of action remains unclear [5].

We also have identified the flagellin N-terminal helical region (COG1344) which includes flagellin and hook associated protein (Aave_4400, XAC1975, Aave_4400, Acav_4298, Acav_4297, Aave_4401) and are reported to form extended helix structures [23]. The HflC (Aave_1431) and HflK (Aave_1432) proteins were assigned to the COG0330 domain, which is the $\mathrm{N}$ terminal of the bacterial membrane proteins. HflK complexes with HflC to form a membrane protease that is modulated by the GTPase HflX [24]. In addition, these proteins are well understood as hypersensitive induced reaction (HIR) proteins and along with prohibitins and stomatins form a PID (proliferation, ion and death) proteins superfamily. These PID proteins share common features in their SPFH (stomatins, prohibitins, flotillins, HflK/C) domains [25]. Toluene tolerance protein (COG2850), which is mediated by an increase in cell membrane rigidity, was also identified. It is a transporter and shows similarity to the $\mathrm{ABC}$ transporter. The RHS protein (COG3209), which contains extended repeat regions that often appear to be involved in ligand binding [26], methyl accepting chemotaxis sensory transducer (COG0840), 3 extracellular solute binding proteins (PFLU1139, Aave_4073, Acav_3681) that was assigned to COG0834, and surface antigen proteins (COG0729) (Table S1). Moreover, 3 general secretary pathway proteins (COG1450), are predicted to be a pore for type III and type IV secretion system used to export proteins across the OM (Table S1).

Thirteen unnamed proteins were also identified in the OM of A. avenae subsp. avenae strain RS-1. These proteins were assigned to families and domain entries of the Pfam database [27]. Acav_1013 and Acav_2514 were assigned to COG0457, while Aave_1831 belongs to TPR (Tetratricopeptide-like repeats) family proteins (GOG4775), which are reported to be involved in the functions such as cell cycle regulation, transcriptional control, mitochondrial and peroxisomal protein transport, neurogenesis and secretion systems [28,29]. Similar to Ralstonia solanacearum, TPR proteins in A. avenae subsp. avenae strain RS-1 may act as type-III-secretion regulators and chaperones [29]. The unnamed protein (Aave_0155), a family of OmpW in COG3047, is known as a family of related proteins, such as porin-like integral membrane proteins [30]. Acav_2307 has no known COG and in Pfam analysis the sequence was notably matched with the integral membrane proteins or lipid anchored proteins of the OmpA family [31]. The unnamed protein with locus tag Aave_4344 was identified as a porin (COG3203) belonging to the general bacterial porin family and acts as a molecular filter for hydrophilic compounds [32]. The unnamed protein Aave_1519 was identified as a universal stress protein (COG0589); Acav_3854, Acav_2307, Acav_4055, and Acav_0287 were assigned to a protein family of unknown function. No COG or Pfam assignment was possible but the deduced proteins carry signal peptides (in silico data not shown). These likely represent examples of new types of OM proteins with functions that are yet to be elucidated.

Of the 63 identified OM proteins, 16 proteins possessed a GRAVY -value $>0$, indicating that some hydrophobic proteins were also identified by this approach [33], including LysR family transcriptional regulator, lipoproteins and an OmpW protein family member (Table S1). A previous study indicated that Gramnegative bacteria have hydrophilic OMs, which act as a barrier against hydrophobic molecules and require transporters to facilitate diffusion into the cytoplasm [34]. Here, we suggest that NodT and OmpW may function as a transporter to facilitate their diffusion into the cells of $A$. avenae subsp. avenae strain RS-1.

In general, in agreement with the result of Tjalsma et al. [35] and Schell et al. [5], our results also revealed the identification of peptides that were recognized as cytoplasmic proteins and cytoplasmic associated proteins, for example, ribosomal proteins, glyoxalase resistance protein/dioxygenase, DNA topoisomerase, elongation factor, tetraacyl disaccharide, ATP synthase subunit beta, Dead/Death box helicase domain-containing, chaperonin GroEL, phosphoglucomutase, ribonuclease E, ribonuclease rng/ rng family, 3-isopropylmalate dehydratase, gluconolactonase and pyruvate kinase. However, most of the proteins in this study identified by LC-MS/MS were OM proteins, lipoproteins and probable $\mathrm{OM}$ proteins (Table $\mathrm{S} 1$ ).

In this comprehensive list of $A$. avenae subsp. avenae strain RS-1 OM proteins and probable OM proteins, many are indispensible for the survival and growth of $A$. avenae subsp. avenae strain RS-1 and accessible on the surface of $A$. avenae subsp. avenae strain RS-1. Therefore, it could be suggested that the depletion of them either could leads to rapid accumulation of unassembled OM proteins in the periplasm followed by cell death as in E. coli [33].

\section{Elucidation and Characterization of in vivo OM Proteome}

As shown in Figure 1, a marked difference was observed in the 1D profile of OM proteins in A. avenae subsp. avenae strain RS-1 between in vitro and in vivo. For LC-MS/MS analysis, peptides released from the in vivo expressed $\mathrm{OM}$ proteome were pooled from two biological replicates. After removing redundant data, the in vivo data were compared with in vitro data, and this indicated that the in vivo expressed OM proteome is different from that found in vitro (Tables S1, S2). Altogether, 62 proteins were obtained in vivo after the comprehensive analysis of two replicates (Table S2), while most of them were lipoproteins, porins, NodT dependent receptors, and TDBR. In addition, in agreement with the result of in vitro experiment, some probable OM proteins like flagellin domain proteins, ABC transporter subunits were also identified in vivo. However, about $7 \%$ of all proteins were also detected as cytoplasmic proteins. The majority of these proteins 
were assigned as ribosomal proteins, DNA topoisomerases and elongation factors.

Overall, results from in vivo study indicated that porin (COG3203), a major OM proteins were generally more populous in vivo than in vitro, which may support the report that porin may play a pivotal role in the bacterial survival in the environment [36]. Therefore, under in vivo conditions, it might be expected that the relative proportion of porins would increase so that the cell could scavenge more successfully for traces of sugars and other carbon sources. Large number of differentially expressed proteins was identified in the OM from strain RS-1 of A. avenae subsp. avenae in in vivo compared to in vitro. Particularly, F proteins (COG1459) which form a platform for the machinery of the type II secretion system (T2SS), as well as the type IV pili and proteins similar to archaeal flagella [37] were not identified in vitro but were identified in vivo (Table 1). In Gram-negative bacteria, there is evidence that T2SS secretes several toxins across the OM [37] and most of the Type II secreted proteins that have been characterized to date are involved in degrading different components of plant cell walls. Therefore, results presented here strongly support this hypothesis that the $\mathrm{F}$ proteins may be involved in the pathogenicity of rice $A$. avenae subsp. avenae pathogen.

Proteins of COG1360 contain an OmpA/MotB domain and are thought to function as porin-like integral membrane proteins or lipid-anchored proteins [38] were identified in vivo (Table 1). The flagellar motor protein MotB and the outer membrane protein OmpA share a region of sequence homology, which describes a domain found fused to T6SS homologues of the T6SS protein DotU, with OmpA/MotB homology. Furthermore, ATPdependent Clp protease (COG0542) was identified only in in vivo condition (Table 1). It belongs to the AAA+super family of ringshaped P-loop NTPases and was associated with diverse cellular activities [39]. Interestingly, a three dimensional fold increase of ClpB matched with the folds of ATP-dependent Clp protease (ClpA) and to chaperone Hsp104 and related ATP-dependent Clp proteases, which belong to T6SS [40].

\section{Functional Differential Expression of the in vivo and in vitro Proteome}

It was established from the LC-MS/MS data that most of proteins were decreased under in vivo conditions compared to in vitro conditions (Table S2), although some unique proteins only expressed under in vivo conditions. The abundance changes of these proteins are provided in (Tables 1, S4). Most of the TonB, NodT and ABC transporter proteins were decreased, indicating that these proteins may be less active under in vivo conditions, but some TonB and ABC transporter proteins, which were also identified but have different sequence homologies compared to in vitro. The family of TonB uses S-adenosylmethionine in the methylation of diverse substrates and the member of this family includes a related group of bacterial proteins of unknown function domains belonging to the periplasmic binding protein superfamily $\mathrm{ABC}$ transporter proteins. In addition, a remarkably high number of TDBR in strain RS-1 of $A$. avenae subsp. avenae under in vitro compared to in vivo is inconsistent with the result of Tsolis et al. [41], who found that TonB dependant proteins contribute to the growth of bacterium in the host (mouse) and indicate the complex functions of these proteins. This could be due to difference of bacterial species and host.

From the lists of OM proteins identified under both in vitro and in vivo conditions, we derived a catalog of shared $\mathrm{OM}$ proteins irrespective of growth conditions. The 48 most abundant of these shared $\mathrm{OM}$ proteins are listed in Table $\mathrm{S} 3$ and represent about $40 \%$ of total OM proteins in the A. avenae subsp. avenae strain RS-1.
Among these shared proteins, the vast majority are porins, TDBR, ABC transporter proteins, flagellin and OmpW-like proteins. The fact that these are abundantly present in cells growing in different growth conditions implies that these shared proteins are fundamental to physiology of $A$. avenae subsp. avenae regardless of the growth conditions. Therefore, these proteins are suggested to represent key elements in controlling detrimental aspects of this bacterium to rice plant, and to exploit the beneficial aspects of this bacterium in plant health.

However, the presence of Protein F, ATP-dependent Clp proteases and OmpA/MotB, as well as some unique flagellin (limited to in vivo condition; Table 1), indicate their importance in bacterial niche adaptation and virulence. Protein F, a component of the type II secretion system (T2SS), is believed to form a membrane complex and serves as a platform on which the pseudopilus is built [42]. The T2SS is a multimeric protein complex and has been reported for pathogenic Gram-negative bacteria to secrete virulence determinants which are commonly toxins and invading the host cells [43]. ATP-dependent proteases have been well understood for their association with T6SS and pathogenicity in Gram-negative bacteria [7]. ATPases are frequently used by secretion systems to energize the transport process, while in the case of the T6SS, a gene encoding ClpB homologue is more frequently found [44]. This was confirmed by a genome wide analysis of $A$. avenae subsp. avenae strain RS-1 in our current study (next section). OmpA is one of the immunodominant antigens and binding of specific anti-OmpA antibodies leads to cell lysis in the presence of complement and can interact with host receptor molecules [45]. MotB (and MotA) serve two functions, the MotA/MotB complex attaches to the cell wall via MotB to form the stator of the flagellar motor, and the MotA/MotB complex couples the flow of ions across the cell membrane to movement of the rotor in most of the Gram-negative bacteria [38].

The association of OmpA/MotB and ATP-dependent Clp proteases with the T6SS make it possible that T6SS may play an important role in survival and pathogencity of $A$. avenae subsp. avenae strain RS-1. Indeed, the T6SS has been identified in $>80$ genomes of Gram-negative bacteria, accounting for about 25\% of currently available bacterial genome sequences. In addition, recent studies have shown that the T6SS has been linked to a variety of functions such as bacterial pathogenicity, host adherence and colonization, cytotoxicity, host-cell invasion, biofilm formation, conjugation, quorum-sensing regulation, survival within macrophages, persistence within the host, both promoting and limiting virulence [46].

\section{Genome Wide Analysis of T6SS Proteins}

It is well known that many bacteria used specialized secretion systems to inject DNA or proteins into organisms like human, animals or plants [47]. However, little is known about the components, location and molecular mechanism of T6SS in bacteria, particularly, plant pathogenic bacteria. Currently, several studies have reported the association of several T6SSs with inner or outer membranes [5,7]. Thus, a genome wide analysis was performed in this study to investigate the association of $\mathrm{OM}$ proteins with T6SS. Using 13 T6SS core components described by Boyer et al. [47] as bait sequences, local BLAST (BLASTN, BLASTX) were used to examine the T6SS locus of $A$. avenae subsp. avenae strain RS-1. T6SS loci were located on three contigs (AAARScontigs 66, AAARScontigs 67 and AAARScontigs 68). For DNA based comparison, three contigs of $A$. avenae subsp. avenae strain RS-1 containing the T6SS were fused and compared to $A$. avenae subsp. citrulli AACOOl and $A$. avenae subsp. avenae 


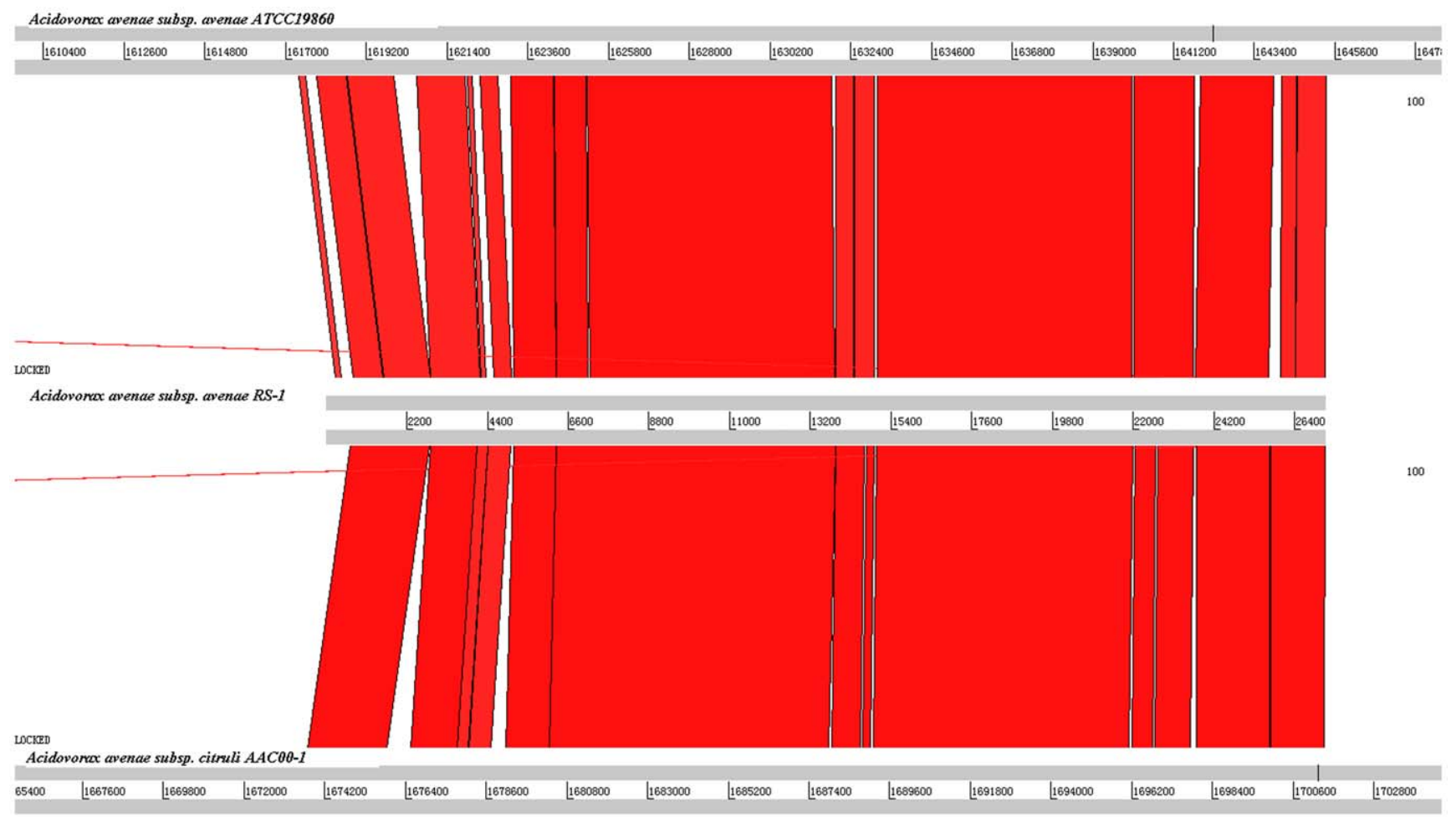

Figure 2. Comparison of the T6SS locus of Acidovorax avenae subsp. avenae strain RS-1 and closely related bacteria by TBLASTN analysis using WebACT and visualized with ACT software.

doi:10.1371/journal.pone.0049657.g002

ATCG19860 using WebAct [48] and visualized by ACT [49] (Fig. 2).

In this study, analysis revealed that only one T6SS gene cluster was found in strain RS-1 of $A$. avenae subsp. avenae encodes T6SS core and conserved accessory components (Fig. 3 and Table S5). As compared to the T6SS of the A. avenae subsp. citrulli, the T6SS locus in $A$. avenae subsp. avenae does not encode either a $\operatorname{VgrG}$ homolog or additional T6SS gene cluster, indicating that this T6SS locus encoded single gene cluster. In addition, bioinformatics analysis of genomic sequence revealed the presence of 12 ORFs encoding putative $\mathrm{VgrG}$ proteins as an orphan component in $A$. avenae subsp. avenae strain RS-1 (Table 2). However, except OmpA/MotB and ClpB, no T6SS protein, even VgrG, was confirmed from LC-MS/MS analysis. One may speculate, if this is the due to their location on the inner membrane or in periplasm.

Interestingly, a genome wide sequence analysis of various bacterial species and strains suggests that the avirulent species often lack a T6SS, whereas pathogenic species often encode orthologs of T6SS as shown for animal pathogens and phytopathogenic bacteria such as Xanthomonas populi and Xanthomonas codiaii [40]. However, orthologs of four hypothetical proteins VCA0118, VCA0119, VCA0121 and VCA0122 were not identified in $A$. avenae subsp. avenae strain RS-1. This is in agreement with the result of Shrivastava and Mande [40]. They showed that bacterial species such as Burkholderia and Xanthomonas lacking one or more of the T6SS orthologs mostly lack orthologs of VCA0118, VGA0119, VCA0121 and VGA0122. This finding indicates that these proteins may be related to T6SS.

Phylogenetic analysis of sequences in this study clearly revealed the association of the predicted T6SS proteins in A. avenae subsp. avenae strain RS-1 with that of the closely related bacteria such as
A. avenae subsp. citrulli strain AAC00-1 and A. avenae subsp. avenae ATCG19860 (Fig. S1).

\section{Conclusions}

In this study, a Triton-insoluble fraction of lysozyme-treated $A$. avenae subsp. avenae strain RS-1 was digested with RNase yielding the purified OM preparation from in vitro and in vivo conditions. Trypsin shaving of $A$. avenae subsp. avenae strain RS-1 OM preparations from in vitro and in vivo growth by LC-MS/MS identified $>159$ OM proteins and probable OM proteins, while in silico functional analysis also predicted their function as $\mathrm{OM}$ or OM-associated (e.g., biosynthesis of EPS, LPS, and cell wall, T6SS, cell division, motility etc). This result revealed that the in vitro vs. in vivo approach developed in this study may also be applied to proteomic comparative analysis of other plant pathogenic bacteria. Furthermore, the comparative global proteomic survey of $A$. avenae subsp. avenae strain RS-1 grown in vitro vs. in vivo revealed differential expression of proteins geared towards survival and pathogenicity of the rice pathogen in host plants. The most dominant proteins induced under in vivo conditions are the secretary proteins, the machinery of the T2SS, type IV pili, T6SS, and the archaeal flagella. In particular, the LC/MS identification of OmpA/MotB and ClpB, validated in silico prediction of T6SS core components based on the sequenced genome of $A$. avenae subsp. avenae strain RS-1. Overall, our in silico functional characterization have most probably confirmed the identified $\mathrm{OM}$ proteins and probable OM proteins based on in vitro and in vivo LC/MS analyses of proteins in A. avenae subsp. avenae strain RS-1. Even though the structure and topology of these machines in depth remain to be fully elucidated. In summary, we made several novel observations in the probable OM localization of T6SS components in particular OmpA/MotB, while several 


\section{Acidovorax avenae strain RS-1 T6SS locus}

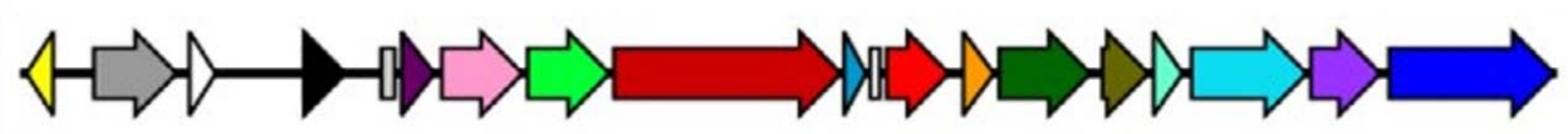

$1 \mathrm{~kb}$

$\longmapsto$

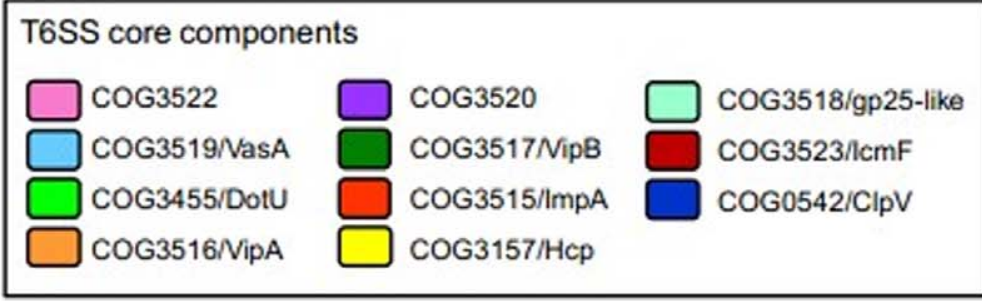

T6SS conserved accesory components

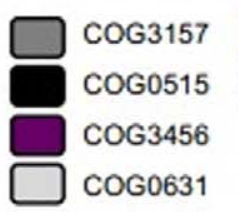

Figure 3. Locus of T6SS core and conserved accessory components in Acidovorax avenae subsp. avenae strain RS-1 which also exist in Acidovorax avenae subsp. citrulli strain AAC00-1 or Burkholderia thailandensis strain E264 are represented with a different color. doi:10.1371/journal.pone.0049657.g003

proteins like protein F, T2SS, porins, OmpA, ATP dependent protease and $\mathrm{ABC}$ transporter proteins may be required for the survival and pathogenicity of $A$. avenae subsp. avenae in host plants.

\section{Materials and Methods}

Bacterial Growth and Culture Conditions

Strain RS-1 of $A$. avenae subsp. avenae was isolated from rice plants in China [1,3] and stored in 20-30\% sterile glycerol (Shanglin Industries, Hangzhou, China) at $-80^{\circ} \mathrm{C}$. For in vitro analysis, the bacterial culture was maintained on fresh Luria Bertani (LB) media plates and incubated at $30^{\circ} \mathrm{C}$ for $24 \mathrm{~h}$. Single colonies were inoculated into $10 \mathrm{~mL}$ of $\mathrm{LB}$ broth and incubated at $30^{\circ} \mathrm{C}$ for overnight. The overnight bacterial cells were re-cultured into $20 \mathrm{~mL}$ fresh LB broth for 6-8 h from initial cell densities of 0.1 O. $D_{600 n m}$ until harvest at late exponential or early stationary

Table 2. Presence of VgrG homologous as an orphan components in Acidovorax avenae subsp. avenae strain RS-1.

\begin{tabular}{lll}
\hline & $\begin{array}{l}\text { VgrG homologs } \\
\text { Serial No. }\end{array}$ & KEGG annotation \\
\hline 1 & Aave_0241 & Rhs element Vgr protein \\
\hline & Aave_0481 & Rhs element Vgr protein \\
3 & Aave_0497 & Rhs element Vgr protein \\
4 & Aave_2047 & Rhs element Vgr protein \\
5 & Aave_2127 & Rhs element Vgr protein \\
6 & Aave_2735 & Rhs element Vgr protein \\
7 & Aave_2840 & Rhs element Vgr protein \\
8 & Aave_3347 & Rhs element Vgr protein \\
9 & Aave_3486 & Rhs element Vgr protein \\
\hline 10 & Aave_3752 & Rhs element Vgr protein \\
11 & Aave_3783 & Rhs element Vgr protein \\
\hline doi:10.1371/journal.pone.0049657.t002 &
\end{tabular}

phase $\left(O . D_{600 n m}\right.$ between 1.8-2.0). Cells were harvested by centrifugation at $8000 \mathrm{rpm}$ for $10 \mathrm{~min}$ and processed for $\mathrm{OM}$ preparation.

For in vivo analysis, bacterial strain was inoculated and recovered as follows. Six days after the inoculation, infected leaves were collected, decontaminated with alcohol. Leaves were cut into pieces with a sterile razor blade and maintained for $20 \mathrm{~min}$ in sterile glass plates containing $20 \mathrm{~mL}$ of distilled water. The incubation during this period of time allowed the bacteria to detach from the leaf tissues. The leaves were separated from the suspension and collected by centrifugation. The bacterial cellpellets were washed with phosphate buffer saline (PBS) and with water and then used for protein extraction.

\section{Preparation of OM Proteins}

Bacterial cells of $A$. avenae subsp. avenae strain RS-1 were collected at late-exponential or early stationary phase from LB broth (referred to as 'in vitro') and from the leaves of $6 \mathrm{~d}$ old infected rice plants (referred to as 'in vivo'), respectively. OM protein extraction was carried out as described earlier [20]. Briefly, the cells were treated with lysozyme $60 \mu \mathrm{g} / \mathrm{mL}$ in the membrane buffer (10 mM Tris, pH 8.0, 0.75 M sucrose, 2 mM EDTA and $1 \mathrm{mM}$ PMSF), disrupted by sonification and centrifuged at $8000 \mathrm{rpm}$ for $10 \mathrm{~min}$ at $4^{\circ} \mathrm{C}$. The supernatant was recovered and centrifuged at $30,000 \mathrm{rpm}$ for $2 \mathrm{~h}$ at $4^{\circ} \mathrm{C}$. Pellets were suspended with $2 \%$ Triton X-100 in the membrane buffer and incubated at room temperature for $30 \mathrm{~min}$ and centrifuged at $30,000 \mathrm{rpm}$ for $2 \mathrm{~h}$ at $4^{\circ} \mathrm{C}$ to isolate the inner membrane proteins. The OM proteins remained in the pellet. The OM protein enriched pellets were suspended in the lysis buffer $(8.5 \mathrm{M}$ urea, $1.98 \mathrm{M}$ thiourea and $2 \%$ CHAPS) and separated by 1D SDSPAGE. The purified OM enriched fraction was treated with RNase and EDTA to remove ribosomal contamination as described by Schell et al. [5].

\section{One-dimensional SDS-PAGE}

Membrane proteins of $A$. avenae subsp. avenae strain RS-1 were separated on a $10 \%$ 1D SDS-PAGE. The samples were analyzed 
according to the method of Laemmli et al. [50] with some modification using an acryl amide concentration of $5 \%$ for the stacking gel and $12 \%$ for the running gel. SDS-PAGE was performed with a mini-gel apparatus (VE-180 vertical electrophoresis bath, Tanon, China). Separated protein bands in the SDSPAGE gel were visualized with both silver staining and Coomassie brilliant blue.

\section{LC-MS/MS of Trypsin-Digested Proteins}

The peptides from purified OM were prepared in two biological replicates and analyzed by LC-MS/MS. Tryptic peptides $(10 \mu \mathrm{L})$ obtained from each band of the SDS-gel of bacterial cell lysate were separated on a Dionex Ultimate 3000 nano-LC system with a Dionex Acclaim PepMap 100, G18 trap column $(20 \mathrm{~mm} \times$ $100 \mu \mathrm{m}, 5 \mu \mathrm{m}, 100 \AA$ ) and Dionex Acclaim PepMap 100, C18 analytical column $(150 \mathrm{~mm} \times 75 \mu \mathrm{m}, 3 \mu \mathrm{m}, 100 \AA)$. The tryptic peptides were loaded onto the trap column using $2 \%$ ACN with $0.025 \%$ TFA at the flow rate of $10 \mu \mathrm{L} / \mathrm{min}$. The enriched tryptic peptides were then separated through the analytical column by gradient elution. The flow rate and column temperature were kept at $250 \mathrm{~nL} / \mathrm{min}$ and $25^{\circ} \mathrm{C}$, respectively. Mobile phases A $(0.1 \%$ formic acid in $5 \%$ acetonitrile) and $\mathrm{B}$ were $0.1 \%$ formic acid in $40 \%$ acetonitrile. The gradient was started at $0 \% \mathrm{~B}$ to $90 \% \mathrm{~B}$ in $40 \mathrm{~min}$. The amaZon ETD ion trap MS equipped with the nano source was used to analyze the tryptic peptides. The scan range for $\mathrm{MS}$ and MS/MS were set at $300-1400 \mathrm{~m} / \mathrm{z}$ and $50-2200 \mathrm{~m} / \mathrm{z}$, respectively. AutoMS/MS was applied with fragmentation at amplitude of $30 \%-200 \%$.

The obtained LC/MS spectra were evaluated by using the MASCOT LC-MS/MS ion search algorithm (Matrix Sciences). All the MS/MS spectra were analyzed by selecting the enzyme trypsin and applying the search parameters of precursor tolerance of $0.35 \mathrm{Da}$ and a fragment tolerance of $0.35 \mathrm{Da}$, oxidation of methionine and carboxyamidomethylation of cysteine were considered as variable and fixed modification, respectively. LCMS/MS profiles of the peptides from $A$. avenae subsp. avenae strain RS-1 were used for searching the sequence similarities to data from Acidovorax species available at NCBI. The cross correlation scores (X corr) [51] of singly-, doubly- and triply-charged peptides were greater than 1.8, 2.5 and 3.5, respectively, were fixed for protein identification. The program listed the peptides corresponding to the proteins. A list of peptide sequences that had the highest $\times$ corr values was identified. Other parameters of $\Delta v a C n>0.1$ are selected for anticipated results in addition to Xcorr score. The proteins were identified either by sufficient number of peptides or identified at least by one peptide that is redundant enough to be considered reliable with acceptable scores. After identifying the proteins with the set threshold values, even the peptides below the defined thresholds were also considered to increase the sequence coverage. The final list of proteins was prepared by combining all the proteins obtained from different LC-MS/MS runs after manual verification. The redundancy of peptides within the list of peptides from each protein was removed by verifying both the $\mathrm{m} / \mathrm{z}$ value and the corresponding sequence. The redundant proteins were also removed.

\section{In silico Analysis of LC-MS/MS Released Peptides}

A genome-wide prediction of protein sub cellular localization has been performed for strain RS-1 of A. avenae subsp. avenae in our previous study [1] using PSORTb (version 3.0.2) and Phobius $[13,14]$ by selecting Gram-negative strains with normal format and at significant score $>7.5$. LC-MS/MS profiles of the peptides from $A$. avenae subsp. avenae strain RS-1 were used for the analysis of the sequence similarities using local BLAST, while complete protein sequences were retrieved from the genome of $A$. avenae subsp. avenae strain RS-1. The grand average of hydropathicity (GRAVY) score of peptides were also evaluated using the ProtParam tool of ExPASy to look at the hydrophobic nature of proteins and SignalP 3.0 server [52] was used to hypothesize Nterminal secretary signal peptides of the identified proteins. Furthermore, proteins annotated by RAST automatic pipeline were used for functional analysis, while locus tag names were assigned from the genome sequenced strain ATCG19860 of $A$. avenae subsp. avenae or more closely related Gram-negative plant pathogenic bacteria. In addition, a list of $A$. avenae subsp. avenae strain RS-1 OM-associated proteins that related with other plant pathogen was also manually assembled by using keywords such as OM, lipoprotein, flagella, pili, secretin, porin, receptor, and surface at Integrated Microbial Genomes (IMG).

\section{In silico Analysis of Type VI Secretion Loci}

A genome wide analysis was carried out to get an overview of T6SS proteins in A. avenae subsp. avenae strain RS-1. Identification of T6SS homologous coding loci were conducted by BLASTN and BLASTX searches using the 13 T6SS core components as baits [47]. For comparative analysis of T6SS, nucleotide sequences were aligned by BLASTN and BLASTX, compared using WebAct online source [49] and visualized by Artemis Comparison Tool (ACT) release 6 [48]. In addition, to identify genes that were represented across A. avenae subsp. avenae strain RS-1, Pseudomonas aeruginosa, Burkholderia mallei, A. avenae subsp. citrulli strain ACC001 and Vibrio cholerae, a phylogenetic profile, which is matrix of the presence/absence of genes across the above bacteria, was created. Phylogenetic tree was built using the maximum likelihood method in MEGA5 [53] with 100 bootstraps. Tree topology was confirmed with the maximum likelihood method using PHYLIP v3.69. After searching, comparing and evaluating the T6SS in A. avenae subsp. avenae strain RS-1, the proteins of T6SS were analyzed for their association with the identified OM proteins regardless of in vivo or in vitro.

\section{Supporting Information}

Figure S1 Evolutionary relationship of T6SS of Acidovorax avenae subsp. avenae strain RS-1. A distance tree (neighbor joining) was calculated from T6SS proteins sequences. Tree topology was confirmed with the maximum likelihood method using PHYLIP v3.69.

(TIF)

Table S1 List of in silico predicated and LC/MS identified in vitro outer membrane protiens of Acidovorax avenae subsp. avenae strain RS-1 proteome. (XLS)

Table S2 List of in silico predicted and LG/MS identified in vivo outer membrane protiens of Acidovorax avenae subsp. avenae strain RS-1 proteome. (XLS)

Table S3 List of LC/MS identified shared outer membrane proteins under in vivo and in vitro of Acidovorax avenae subsp. avenae strain RS-1 proteome.

(XLS)

Table S4 List of LG-MS/MS identified unique outer membrane proteins under in vitro condition of Acidovorax avenae subsp. avenae strain RS-1 proteome. (XLS) 
Table S5 T6SS gene cluster including outer membrane proteins OmpA/MotB domain in Acidovorax avenae subsp. avenae strain RS-1 genome.

(XLS)

\section{Acknowledgments}

We are great thankful to Prof Guanwu Li, Proteomic Laboratory for Drug Discovery, Shantou University Medical College, Guangdong, China for providing the technical assistance in LC-MS/MS analysis.

\section{References}

1. Xie GL, Zhang GQ Liu H, Lou MM, Tian WX, et al. (2011) Genome sequence of the rice pathogenic bacterium Acidovorax avenae subsp. avenae RS-1. J Bacteriol. 193: 5013-5014

2. Xie GL, Sun XL, Mew TW (1998) Characterization of Acidovorax avenae subsp. avenae from Rice Seeds. Chin J Rice Sci. 12: 165-171.

3. Li B, Liu B, Yu R, Tao Z, Wang Y, et al. (2011) Bacterial brown stripe of rice in soil-less culture system caused by Acidovorax avenae subsp. avenae in China. J Gen Plant Pathol. 77: 64-67.

4. Kuehn MJ, Kesty NC (2005) Bacterial outer membrane vesicles and the hostpathogen interaction. Gen Dev. 19: 2645-2655.

5. Schell MA, Zhao P, Wells L (2011) Outer membrane proteome of Burkholderia pseudomallei and Burkholderia mallei from diverse growth conditions. J Proteome Res. 10: 2417-2424.

6. Aschtgen MS, Bernard CS, De Bentzmann S, Lloubes R, Cascales E (2008) SciN is an outer membrane lipoprotein required for type VI secretion in enteroaggregative Escherichia coli. J Bacteriol. 190: 7523-7531.

7. Pieper R, Huang ST, Clark DJ, Robinson JM, Parmar PP, et al. (2008) Characterizing the dynamic nature of the Yersinia pestis periplasmic proteome in response to nutrient exhaustion and temperature change. Proteomics 8: 14421458.

8. Sidhu VK, Vorhoelter FJ, Niehaus K, Watt SA (2008) Analysis of outer membrane vesicle associated proteins isolated from the plant pathogenic bacterium Xanthomonas campestris pv. campestris. BMC Microbiol. 8: 87.

9. Li G, Howard SP (2010) ExeA binds to peptidoglycan and forms a multimer for assembly of the type II secretion apparatus in Aeromonas hydrophila. Mol Microbiol. 76: 772-781.

10. D'Alessandro B, Lery LMS, von Krueger WMA, Lima A, Piccini C, et al. (2011) Proteomic analysis of Proteus mirabilis outer membrane proteins reveals differential expression in vivo vs. in vitro conditions. FEMS Immunol Med Microbiol. 63: 174-182.

11. Knief C, Delmotte N, Vorholt JA (2011) Bacterial adaptation to life in association with plants - A proteomic perspective from culture to in situ conditions. Proteomics 11: 3086-3105.

12. Cash P (2011) Investigating pathogen biology at the level of the proteome. Proteomics 11: 3190-3202.

13. Kall L, Krogh A, Sonnhammer ELL (2004) A combined transmembrane topology and signal peptide prediction method. J Molecul Biol. 338: 1027-1036.

14. Yu NY, Wagner JR, Laird MR, Melli G, Rey S, et al. (2010) PSORTb 3.0: improved protein subcellular localization prediction with refined localization subcategories and predictive capabilities for all prokaryotes. Bioinformatics 26 : 1608-1615.

15. Bairoch A, Apweiler R (2000) The SWISS-PROT protein sequence database and its supplement TrEMBL in 2000. Nucleic Acids Res. 28: 45-48.

16. Blanvillain S, Meyer D, Boulanger A, Lautier M, Guynet C, et al. (2007) Plant carbohydrate scavenging through TonB-dependent receptors: A feature shared by phytopathogenic and aquatic bacteria. PLoS one 2: e224.

17. Buchanan SK (2001) Type I secretion and multidrug efflux: transport through the TolC channel-tunnel. Tren Biochem Sci. 26: 3-6.

18. Babujee L, Venkatesh B, Yamazaki A, Tsuyumu S (2007) Proteomic analysis of the carbonate insoluble outer membrane fraction of the soft-rot pathogen Dickeya dadantii (syn. Enwinia chrysanthemi) strain 3937. J Proteome Res. 6: 62-69.

19. Chung JW, Ng-Thow-Hing C, Budman LI, Gibbs BF, Nash JHE, et al. (2007) Outer membrane proteome of Actinobacillus pleuropneumoniae: LC-MS/MS analyses validate in silico predictions. Proteomics 7: 1854-1865.

20. Jagannadham MV (2008) Identification of proteins from membrane preparations by a combination of MALDI TOF-TOF and LC-coupled linear ion trap MS analysis of an Antarctic bacterium Pseudomonas syringae Lz4W, a strain with unsequenced genome. Electrophoresis 29: 4341-4350.

21. Hossain MJ, Rahman KS, Terhune JS, Liles MR (2012) An outer membrane porin protein modulates phage susceptibility in Edwardsiella ictaluri. MicrobiolSGM 158: 474-487.

22. Akama H, Kanemaki M, Yoshimura M, Tsukihara T, Kashiwagi T, et al. (2004) Crystal structure of the drug discharge outer membrane protein, OprM, of Pseudomonas aeruginosa - Dual modes of membrane anchoring and occluded cavity end. J Biol Chem. 279: 52816-52819.

23. DeRosier DJ (1995) Spinning tails. Curr Opinion Struct Biol. 5: 187-193.

24. Noble JA, Innis MA, Koonin EV, Rudd KE, Banuett F, et al. (1993) The Escherichia-coli hfla locus encodes a putative gtp-binding protein and 2

\section{Author Contributions}

Conceived and designed the experiments: BL GLX YLW GCS. Performed the experiments: MI YS. Analyzed the data: MI YS HQ AJ LPL HL. Contributed reagents/materials/analysis tools: BL GLX YLW GCS. Wrote the paper: MI BL MK.

membrane-proteins, one of which contains a protease-like domain. Proc Natl Acad Sci USA 90: 10866-10870.

25. Choi HW, Kim YJ, Hwang BK (2011) The hypersensitive induced reaction and leucine-rich repeat proteins regulate plant cell death associated with disease and plant immunity. Mol Plant Microbe Int. 24: 68-78.

26. Minet AD, Chiquet ER (2000) Phylogenetic analysis of teneurin genes and comparison to the rearrangement hot spot elements of E. coli. Gene 257: 87-97.

27. Punta M, Coggill PC, Eberhardt RY, Mistry J, Tate J, et al. (2012) The Pfam protein families database. Nucleic Acid Res. 40: D290-D301.

28. Das AK, Cohen PTW, Barford D (1998) The structure of the tetratricopeptide repeats of protein phosphatase 5: implications for TPR-mediated protein-protein interactions. EMBO J. 17: 1192-1199.

29. Pallen MJ, Chaudhuri RR, Henderson IR (2003) Genomic analysis of secretion systems. Curr Opinion Microbiol. 6: 519-527.

30. Pilsl H, Smajs D, Braun V (1999) Characterization of colicin S4 and its receptor, OmpW, a minor protein of the Escherichia coli outer membrane. J Bacteriol. 181: 3578-3581.

31. Selvaraj V, Buttke DE, Asano A, McElwee JL, Wolff CA, et al. (2007) G(M1) dynamics as a marker for membrane changes associated with the process of capacitation in murine and bovine spermatozoa. J Androl. 28: 588-599.

32. Benz R, Bauer K (1988) Permeation of hydrophilic molecules through the outermembrane of gram-negative bacteria - review on bacterial porins. Eur J Biochem. 176: 1-19.

33. Ricci DP, Hagan CL, Kahne D, Silhavy TJ (2012) Activation of the Escherichia coli $\beta$-barrel assembly machine (Bam) is required for essential components to interact properly with substrate. Proc Natl Acad Sci USA 109: 3487-3491.

34. van den Berg B (2005) The FadL family: unusual transporters for unusual substrates. Curr Opinion Struct Biol. 15: 401-407.

35. Tjalsma H, Lambooy L, Hermans PW, Swinkels DW (2008) Shedding and shaving: Disclosure of proteomic expressions on a bacterial face. Proteomics 8: 1415-1428.

36. Ozkanca R, Flint KP (2002) The effect of starvation stress on the porin protein expression of Escherichia coli in lake water. Lett App Microbiol. 35: 533-537.

37. Peabody CR, Chung YJ, Yen MR, Vidal-Ingigliardi D, Pugsley AP, et al. (2003) Type II protein secretion and its relationship to bacterial type IV pili and archaeal flagella. Microbiol-SGM 149: 3051-3072.

38. Mougous JD, Cuff ME, Raunser S, Shen A, Zhou M, et al. (2006) A virulence locus of Pseudomonas aeruginosa encodes a protein secretion apparatus. Science 312: $1526-1530$.

39. Snider J, Thibault G, Houry WA (2008) The AAA plus superfamily of functionally diverse proteins. Gen Biol. 9: 216.

40. Shrivastava S, Mande SS (2008) Identification and Functional Characterization of Gene Components of Type VI Secretion System in Bacterial Genomes. PloS one 3: 2955.

41. Tsolis RM, Baumler AJ, Heffron F, Stojiljkovic I (1996) Contribution of TonBand Feo-mediated iron uptake to growth of Salmonella typhimurium in the mouse. Infect Immun. 64: 4549-4556.

42. Py B, Loiseau L, Barras F (2001) An inner membrane platform in the type II secretion machinery of Gram-negative bacteria. EMBO Reports 2: 244-248.

43. Baldi DL, Higginson EE, Hocking DM, Praszkier J, Cavaliere R, et al. (2012) The type II secretion system and its ubiquitous lipoprotein substrate, SslE, are required for biofilm formation and virulence of enteropathogenic Escherichia coli. Infect Immun. 80: 2042-2052.

44. Filloux A, Hachani A, Bleves S (2008) The bacterial type VI secretion machine: yet another player for protein transport across membranes. Microbiol-SGM 154: $1570-1583$.

45. Dumetz F, LaPatra SE, Duchaud E, Claverol S, Le Henaff M (2007) The Flavobacterium psychrophilum OmpA, an outer membrane glycoprotein, induces a humoral response in rainbow trout. J Appl Microbiol. 103: 14611470 .

46. Cotter P (2011) Microbiology molecular syringes scratch the surface. Nature 475: 301-303

47. Boyer F, Fichant G, Berthod J, Vandenbrouck Y, Attree I (2009) Dissecting the bacterial type VI secretion system by a genome wide in silico analysis: what can be learned from available microbial genomic resources? BMC Genomics 10: 104.

48. Carver TJ, Rutherford KM, Berriman M, Rajandream MA, Barrell BG, et al. (2005) ACT: the Artemis comparison tool. Bioinformatics 21: 3422-3423. 
49. Abbott JC, Aanensen DM, Rutherford K, Butcher S, Spratt BG (2005) WebACT - an online companion for the artemis comparison tool. Bioinformatics 21: 3665-3666.

50. Laemmli UK, Molbert E, Showe M, Kellenbe E (1970) Form-determining function of genes required for assembly of head of bacteriophage-t4. J Mol Biol. 49: 99-113.

51. Olsen JV, de Godoy LMF, Li GQ, Macek B, Mortensen P, et al. (2005) Parts per million mass accuracy on an orbitrap mass spectrometer via lock mass injection into a C-trap. Mol Cell Proteomics 4: 2010-2021.
52. Petersen TN, Brunak S, von Heijne G, Nielsen H (2011) SignalP 4.0: discriminating signal peptides from transmembrane regions. Nat Methods 8: 785-786.

53. Tamura K, Peterson D, Peterson N, Stecher G, Nei M, et al. (2011) MEGA5: Molecular evolutionary genetics analysis using maximum likelihood, evolutionary distance, and maximum parsimony methods. Mol Biol Evol. 28: 2731-2739. 